\title{
CURVATURE OPERATORS AND CHARACTERISTIC CLASSES
}

\author{
BY
}

IRL BIVENS

\begin{abstract}
Given tensors $A$ and $B$ of type $(k, k)$ on a Riemannian manifold $M$ we construct in a natural way a $2 k$ form $F_{k}(A, B)$. If $A$ and $B$ satisfy the generalized Codazzi equations then this $2 k$ form is closed. In particular if $R_{2 k}$ denotes the $2 k$ th curvature operator then $F_{2 k}\left(R_{2 k}, R_{2 k}\right)$ is (up to a constant multiple) the $k$ th Pontrjagin class of $M$. By means of a theorem of Gilkey we give conditions sufficient to guarantee that a form constructed from more complicated expressions involving the curvature operators does in fact belong to the Pontrjagin algebra. As a corollary we obtain Thorpe's vanishing theorem for manifolds with constant $2 p$ th sectional curvature.

If at each point in $M$ the tangent space contains a subspace of a particular type (similar to curvature nullity) we show that certain Pontrjagin classes must vanish. We generalize the result that submanifolds of Euclidean space with flat normal bundle have a trivial Pontrjagin algebra.

The curvature operator, $R_{2}$, is interesting in that the components of $\boldsymbol{R}_{\mathbf{2}}$ with respect to any orthonormal frame are given by certain universal (independent of frame) homogeneous linear polynomials in the components of the curvature tensor. We characterize all such operators and using this characterization derive in a natural way the Weyl component of $\boldsymbol{R}_{\mathbf{2}}$.
\end{abstract}

1. Introduction. Let $S$ be a tensor of type $(k, k)$ on a Riemannian manifold $(M,\langle\rangle$,$) which is alternating in the first k$ and in the last $k$ indices. At each point $x \in M, S_{x}$ may be identified with an element of End $\Lambda^{k} T_{x} M$ and we write $S \in \Gamma\left[\right.$ End $\left.\Lambda^{k}(T M)\right]$. Given $S \in \Gamma\left[\right.$ End $\left.\Lambda^{k}(T M)\right]$ and $T \in \Gamma\left[\right.$ End $\left.\Lambda^{j}(T M)\right]$ we may define $S * T \in \Gamma\left[\right.$ End $\left.\Lambda^{k+j}(T M)\right]$ by wedging the respective covariant and contravariant components of $S$ and $T$. The multiplication, *, is associative and commutative and we have the following formula

$$
\begin{aligned}
S * T\left(X_{1} \wedge X_{2} \wedge \cdots \wedge X_{k+j}\right)=\frac{1}{k ! j !} \sum \varepsilon(\sigma) S\left(X_{\sigma(1)} \wedge \cdots \wedge X_{\sigma(k)}\right) \\
\wedge T\left(X_{\sigma(k+1)} \wedge \cdots \wedge X_{\sigma(k+j)}\right),
\end{aligned}
$$

where the sum is over all elements of the symmetric group $S_{k+j}$. Let $\nabla$ denote the Riemannian connection on the full tensor algebra of $M$.

Definition 1.1. A Codazzi tensor of type $(k, k)$ will be an element $S$ of $\Gamma\left[\right.$ End $\left.\Lambda^{k}(T M)\right]$ such that

$$
0=\sum(-1)^{j+1}\left(\nabla_{X_{j}} S\right)\left(X_{1} \wedge X_{2} \wedge \cdots \wedge X_{j-1} \wedge X_{j+1} \wedge \cdots \wedge X_{k+1}\right)
$$

for all $C^{\infty}$ vector fields $X_{1}, X_{2}, \ldots, X_{k+1}$ on $M$.

Received by the editors October 20, 1980.

1980 Mathematics Subject Classification. Primary 57R20, 53A45; Secondary 58A12, 58A10. 
A routine computation shows that if $S$ and $T$ are Codazzi tensors of types $(k, k)$ and $(j, j)$ respectively then $S * T$ is a Codazzi tensor of type $(k+j, k+j)$.

The curvature operators $R_{2 k} \in \Gamma\left[\right.$ End $\left.\Lambda^{2 k}(T M)\right]$ are defined by $\left\langle R_{2}(X \wedge Y), Z\right.$ $\wedge W\rangle=\langle R(X, Y) Z, W\rangle$ and $R_{2 k}=R_{2} * R_{2} * \cdots * R_{2}$ ( $k$ times), where $R$ denotes the curvature tensor of $M$. The second Bianchi identity implies $R_{2}$ is a Codazzi tensor of type $(2,2)$ and consequently $R_{2 k}$ is a Codazzi tensor of type $(2 k, 2 k)$. As in $[7,9,10]$ the notion of the first Bianchi identity may be extended via

Definition 1.2. An element $S$ of $\Gamma\left[\right.$ End $\left.\Lambda^{k}(T M)\right]$ is said to satisfy the Bianchi identity provided

$$
0=\sum \varepsilon(\sigma)\left\langle S\left(X_{\sigma(1)} \wedge \cdots \wedge X_{\sigma(k)}\right), X_{\sigma(k+1)} \wedge X_{k+2} \wedge \cdots \wedge X_{2 k}\right\rangle,
$$

where the sum is over all elements $\sigma$ of the symmetric group $S_{k+1}$ and $X_{1}, X_{2}, \ldots, X_{2 k}$ are any $C^{\infty}$ vector fields on $M$.

An element $S$ of $\Gamma\left[\right.$ End $\left.\Lambda^{1}(T M)\right] \equiv \Gamma[\operatorname{End}(T M)]$ satisfies the Bianchi identity if and only if $S$ is symmetric. If $k=2$ and $S=R_{2}$ then (2) reduces to the first Bianchi identity for the curvature tensor $R$. Since the space of tensors satisfying (2) is closed under $*$ it follows that the curvature operators $R_{2 k}$ satisfy the Bianchi identity.

Given $A, B \in \Gamma\left[\right.$ End $\left.\Lambda^{k}(T M)\right]$ we may define a $2 k$ form $F_{k}(A, B)$ by the formula

$$
\begin{aligned}
F_{k}(A, B) & \left(X_{1}, X_{2}, \ldots, X_{2 k}\right) \\
= & \sum \varepsilon(\sigma)\left\langle A\left(X_{\sigma(1)} \wedge \cdots \wedge X_{\sigma(k)}\right), B\left(X_{\sigma(k+1)} \wedge \cdots \wedge X_{\sigma(2 k)}\right)\right\rangle
\end{aligned}
$$

where the sum is over $S_{2 k}$ and $X_{1}, X_{2}, \ldots, X_{2 k}$ are $C^{\infty}$ vector fields on $M$. If $A$ and $B$ are Codazzi tensors of type $(k, k)$ then the $2 k$ form $F_{k}(A, B)$ is closed. In particular $F_{2 k}\left(R_{2 k}, R_{2 k}\right)$ is a closed $4 k$ form on $M$ and in fact $F_{2 k}\left(R_{2 k}, R_{2 k}\right)$ is (up to a constant multiple) the deRham representative of the $k$ th Pontrjagin class $P_{k}$ of $M[2,9]$. It follows that assumptions about the curvature operators may imply the vanishing of particular Pontrjagin classes. For example, a Riemannian manifold has constant sectional curvature if and only if the curvature operator, $R_{2}$, is a scalar multiple of the identity. An immediate corollary is Chern's result that a manifold with constant sectional curvature has a trivial Pontrjagin algebra [2].

It is natural to ask what can be said about forms constructed from more complicated expressions involving the curvature operators (e.g., $\left.F_{4}\left[\left(R_{2} \circ R_{2}\right) * R_{2}, R_{4}\right]\right)$. In Theorem 2.3 we give sufficient conditions for such forms to lie in the Pontrjagin algebra and derive as a corollary Thorpe's vanishing theorem for manifolds with constant $2 p$ sectional curvature [10]. The proof of Theorem 2.3 relies heavily on Gilkey's theorem [1] which states that a particular class of transformations mapping the set of Riemannian metrics on $M$ to the space of $q$-forms on $M$ must in fact have values in the Pontrjagin algebra.

In [6] Gray generalized the notion of curvature nullity [3] and defined for a given integer $q$, a particular subspace $N_{m}$ of $T_{m} M$. Under the assumption that $\mu=$ $\operatorname{dim} N_{m}$ is constant Gray showed that $P_{k}=0$ if $k \geqslant \frac{1}{2}(q+n-\mu), n=\operatorname{dim} M$. If 
we relax the assumption that $\operatorname{dim} N_{m}$ is constant and redefine $\mu$ to be the minimum dimension of $N_{m}$ then

Proposition 2.5. If $k \geqslant q / 2$ with $2 k \equiv r \bmod q, 0 \leqslant r<q$ and $k-r\rangle$ $(n-\mu) / 4$ then $P_{k}=0$.

It then follows as a corollary that $P_{k}=0$ for $k \geqslant \frac{1}{2}(q+n-\mu)$.

In Proposition 2.7 we generalize the well-known result that a submanifold of Euclidean space with flat normal bundle has a trivial Pontrjagin algebra. The proofs of both Proposition 2.5 and Proposition 2.7 are "point-wise" in that the $4 k$ forms $F_{2 k}\left(R_{2 k}, R_{2 k}\right)$ (for appropriate choices of $k$ ) are shown to be identically zero.

The curvature operator $R_{2}$ is interesting in that the components of $R_{2}$ with respect to any orthonormal frame are given by certain universal (independent of frame) homogeneous linear polynomials in the components of the curvature tensor. The same is true for the tensors $T_{1}, T_{2}$ of type $(1,1)$ defined by $\left\langle T_{1}(X), Y\right\rangle=$ $\operatorname{Ric}(X, Y)$ and $T_{2}=\tau I$ where Ric denotes the Ricci tensor, $\tau$ the scalar curvature and $I$ denotes the tensor of type $(1,1)$ which at each point of $M$ is the identity endomorphism. Any element $B$ of $\Gamma\left[\right.$ End $\left.\Lambda^{k}(T M)\right]$ which has this property we call a linear polynomial operator and we have the following characterization.

THEOREM 3.1. Let $B$ be a linear polynomial operator in $\Gamma\left[\right.$ End $\left.\Lambda^{k}(T M)\right]$.

(a) If $k=1, B=a T_{1}+b T_{2}, a, b \in \mathbf{R}$;

(b) if $k \geqslant 2, B=\left(a T_{1}+b T_{2}\right) * I_{k-1}+c R_{2} * I_{k-2}, a, b, c \in \mathbf{R}$, where $I_{r}$ denotes the identity element of $\Gamma\left[\right.$ End $\left.\Lambda^{r}(T M)\right]$.

The decomposition of the curvature operator $R_{2}$ (and in particular the expression for the Weyl component) given in [8] follows in a natural way from Theorem 3.1.

2. The Pontrjagin classes. In [2] Chern showed that up to a constant factor the $k$ th Pontrjagin class $P_{k} \in H^{4 k}(M, R)$ is represented by the $4 k$ form $F_{2 k}\left(R_{2 k}, R_{2 k}\right)$. (The formulation in terms of the curvature operators is due to Stehney [9].) In this section we consider assumptions about the curvature operators which force the vanishing of particular Pontrjagin classes.

Lemma 2.1. (a) Let $B \in \Gamma\left[\right.$ End $\left.\Lambda^{k}(T M)\right], A \in \Gamma\left[\right.$ End $\left.\Lambda^{k-1}(T M)\right]$ and $I \equiv I_{1} \in$ $\Gamma[\operatorname{End}(T M)]$. If $B$ satisfies the Bianchi identity then $F_{k}(B, A * I)=0$.

(b) Let $B_{1}, B_{2} \in \Gamma\left[\right.$ End $\left.\Lambda^{k}(T M)\right]$. Suppose $\left\{e_{1}, e_{2}, \ldots, e_{n}\right\}$ is an orthonormal basis for $T_{x} M$ such that $\left\{e_{L_{1}} \wedge e_{L_{2}} \wedge \cdots \wedge e_{L_{k}} \mid L_{1}<L_{2}<\cdots<L_{k}\right\}$ is a basis of eigenvectors for $B_{1}$ and $B_{2}$ at $x$. If $A_{1}, A_{2} \in \Gamma\left[\right.$ End $\left.\Lambda^{r}(T M)\right]$ for $0 \leqslant r<k$ then at $x$, $F_{k+r}\left(B_{1} * A_{1}, B_{2} * A_{2}\right)=0$.

Proof. (a) Routine.

(b) It suffices to show $F_{k+r}\left(B_{1} * A_{1}, B_{2} * A_{2}\right)\left(e_{L_{1}}, e_{L_{2}}, \ldots, e_{L_{2(k+r)}}\right)=0$ for $L_{1}<L_{2}$ $<\cdots<L_{2(k+r)}$. This term is a linear combination of expressions of the form $\left\langle e_{S_{1}} \wedge \cdots \wedge e_{S_{k}} \wedge X, e_{j_{1}} \wedge \cdots \wedge e_{j_{k}} \wedge Y\right\rangle$ where $X, Y \in \Lambda^{r} T_{x} M$ and 
$\left\{e_{S_{1}}, \ldots, e_{S_{k}}, e_{j_{1}}, \ldots, e_{j_{k}}\right\}$ is a set of distinct orthonormal vectors. The value of such an expression is given by the determinant of a matrix of the form

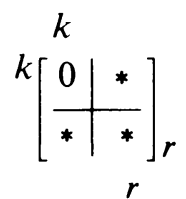

This matrix is singular for $0 \leqslant r<k$, and the result follows.

As an application of these ideas suppose $M$ is a hypersurface of a space of constant sectional curvature $c$. Let $N$ be a local unit normal vector field on $M$ and define an associated local tensor, $S$, of type $(1,1)$ on $M$ by $S(X)=-\tilde{\nabla}_{X} N$, where $\tilde{\nabla}$ denotes covariant differentiation in the ambient space. $S$ is symmetric and the Gauss curvature equations become $R_{2}=c I_{2}+\frac{1}{2} S * S$, where $I_{2}$ denotes the identity. It follows that locally $R_{2 k}$ is a linear combination of terms of the form $I_{2}^{k-r} * S^{2 r}$ (where the powers denote star multiplication), $r=0, \ldots, k$. Using Lemma 2.1 and the bilinearity of $F_{2 k}$ we conclude that $F_{2 k}\left(R_{2 k}, R_{2 k}\right) \equiv 0, k \geqslant 1$. Consequently $M$ has a trivial Pontrjagin algebra. A similar argument using (b) of Lemma 2.1 yields the following result $[4,9]$.

PROPOSITION 2.2. If $R_{2 k}=c S^{2 k}$ where $c \in \mathbf{R}$ and $S$ is a symmetric tensor of type $(1,1)$ then $P_{q}=0$ for $q \geqslant k$.

RemarK. For $k=1$ this result is due to Chern [2].

A geometric object $w(g)$ associated with a Riemannian metric, $g$, on a manifold, $M$, is said to have weight $k$ provided $w\left(c^{2} g\right)=c^{k} w(g)$ for $c$ any positive real number. Suppose we have a function which associates to a Riemannian manifold, $(M, g)$, a $p$ form $w(M, g)$. Further suppose that the components of $w(M, g)$, relative to any orthonormal frame, are given by certain universal (independent of frame) polynomials in the components of the curvature tensor with respect to the given frame. If $w(M, g)$ has weight $k \geqslant 0$ then Gilkey's Theorem [1] states that $w(M, g)$ belongs to the Pontrjagin algebra of $M$ and is zero for $k>0$. With the aid of this result we now consider forms constructed from more complicated expressions involving the curvature operators.

Definition 2.1. $B_{2 k}^{i}=R_{2 k} \circ R_{2 k} \circ \cdots \circ R_{2 k}$ ( $i$ times), $B_{2 k}^{0}=$ identity.

Definition 2.2. Let $I=\left(i_{1}, \ldots, i_{s}\right), K=\left(k_{1}, \ldots, k_{s}\right)$ be multi-indices of nonnegative integers with

(a) $C_{K}^{I}=B_{2 k_{1}}^{i_{1}} \cdots * B_{2 k_{s}}^{i_{s}}$,

(b) $|K|=\sum k_{j}$,

(c) $I \cdot K=\sum i_{j} k_{j}$.

THEOREM 2.3. Consider $F_{m}\left(C_{K}^{I}, C_{L}^{J}\right)$ where $m=2|K|=2|L|$. If $m \geqslant I \cdot K+J \cdot L$ then $F_{m}\left(C_{K}^{I}, C_{L}^{J}\right)$ is in the Pontrjagin algebra and is zero for $m>I \cdot K+J \cdot L$.

Proof. It is routine to verify that the components of $F_{m}\left(C_{K}^{I}, C_{L}^{J}\right)$ are given by universal polynomials in the components of the curvature tensor (with respect to an orthonormal frame). $R_{2}$ has weight -2 and consequently $R_{2 k}$ has weight $-2 k, B_{2 k}^{i}$ 
has weight $-2 k i$, and $C_{K}^{I}$ has weight $-2 I \cdot K$. Since $F_{m}$ has weight $2 m$ the weight of $F_{m}\left(C_{K}^{I}, C_{L}^{J}\right)$ is $2(m-I \cdot K-J \cdot L)$ and the result follows.

A manifold has constant $2 k$ sectional curvature in the sense of [10] if and only if $R_{2 k}$ is a scalar multiple of the identity. As a corollary of Theorem 2.3 we have the following result of Thorpe [10].

Corollary 2.4. If $M$ has constant $2 k$ sectional curvature then $P_{q}=0$ for $q \geqslant k$.

Proof. If $M$ has constant $2 k$ sectional curvature then $R_{2 k}=c I_{2 k}$ for some constant $c$. Let $m=2(k+r)$.

$$
F_{m}\left(R_{m}, R_{m}\right)=F_{m}\left(R_{2 k} * R_{2 r}, R_{2 k} * R_{2 r}\right)=c^{2} F_{m}\left(B_{2 k}^{0} * B_{2 r}^{1}, B_{2 k}^{0} * B_{2 r}^{1}\right) \text {. }
$$

Since $m>0 k+1 r+0 k+1 r, P_{q}=0$ for $q \geqslant k$.

Proposition 2.2 is one generalization of Thorpe's result. We now give another. Fix an even positive integer $q$ and a real number $c$.

Definition 2.3. $N_{m}=\left\{x_{1} \in T_{m} M: R_{q}\left(x_{1} \wedge x_{2} \wedge \cdots \wedge x_{q}\right)=c x_{1} \wedge x_{2}\right.$ $\wedge \cdots \wedge x_{q}$ for all $\left.x_{2}, \ldots, x_{q} \in T_{m} M\right\}$.

Gray [6] showed that if the dimension of $N_{m}$ is a constant, $\mu$, then the distribution, $m \rightarrow N_{m}$, is integrable with totally geodesic leaves. Using this fact he then proved that $P_{k}=0$ for $k \geqslant \frac{1}{2}(q+n-\mu), n=\operatorname{dim} M$. Suppose now that the dimension $N_{m}$ is not necessarily constant and let $\mu=\min _{m} \operatorname{dim} N_{m}$.

Proposition 2.5. If $k \geqslant q / 2$ with $2 k \equiv r \bmod q, 0 \leqslant r<q$ and $k-r\rangle$ $(n-\mu) / 4$ then $P_{k}=0$.

Proof. Fix a point $p \in M$, let $h=\operatorname{dim} N_{p}$ and let $W$ denote the orthogonal complement of $N_{p}$. Choose an orthonormal frame, $e_{1} \cdots e_{n}$, at $p$ with $e_{1}, \ldots, e_{h}$ an orthonormal basis for $N_{p}$. Write $2 k=j q+r$ for $j \geqslant 1$ and note that $j q-$ $(n-\mu) / 2>r$. To show $P_{k}=0$ it suffices to show

$$
\left\langle R_{2 k}\left(e_{i_{1}} \wedge \cdots \wedge e_{i_{2 k}}\right), \quad R_{2 k}\left(e_{i_{2 k+1}} \wedge \cdots \wedge e_{i_{4 k}}\right)\right\rangle=0
$$

for any choice of distinct indices $i_{1}, \ldots, i_{4 k}$. Since the dimension of $W$ is $n-h$, we may assume without loss of generality that there are $m \leqslant(n-\mu) / 2$ elements of $W$ in the set $\left\{e_{i_{1}}, \ldots, e_{i_{2 k}}\right\}$. Since $R_{2 k}$ is selfadjoint

$$
\begin{gathered}
\left\langle R_{2 k}\left(e_{i_{1}} \wedge \cdots \wedge e_{i_{2 k}}\right), R_{2 k}\left(e_{i_{2 k+1}} \wedge \cdots \wedge e_{i_{4 k}}\right)\right\rangle \\
=\left\langle R_{2 k} \circ R_{2 k}\left(e_{i_{1}} \wedge \cdots\right), e_{i_{2 k+1}} \wedge \cdots\right\rangle .
\end{gathered}
$$

Up to a constant factor $R_{2 k}\left(e_{i_{1}} \cdots e_{i_{2 k}}\right)$ is equal to

$$
\begin{aligned}
\sum \varepsilon(\sigma) R_{q}\left(e_{i_{\sigma(1)}} \wedge \cdots \wedge e_{i_{\sigma}(q)}\right) & \wedge \cdots \wedge R_{q}\left(\cdots \wedge e_{i_{\sigma(j q)}}\right) \\
& \wedge R_{r}\left(e_{i_{\sigma}(j q+1)} \wedge \cdots \wedge e_{i_{\sigma(2 k)}}\right) .
\end{aligned}
$$

Now the number, $s$, of elements of $N_{p}$ in the set, $\left\{e_{i_{\sigma(1)}} \cdots e_{i_{\sigma(q q)}}\right\}$, is greater than $r$ since $s \geqslant j q-m \geqslant j q-(n-\mu) / 2>r$. Thus up to a constant factor $R_{2 k}\left(e_{i_{1}} \wedge \cdots \wedge e_{i_{2 k}}\right)$ is a sum of terms of the form $z_{1} \wedge \cdots \wedge z_{2 k}$ where there are at least $r+1$ elements of $\left\{e_{i_{1}}, \ldots, e_{i_{2 k}}\right\} \cap N_{p}$ in the set $\left\{z_{1}, \ldots, z_{2 k}\right\}$. But then by the same decomposition above $R_{2 k}\left(z_{1} \wedge \cdots \wedge z_{2 k}\right)$ must be a sum of terms of the 
form $x_{1} \wedge \cdots \wedge x_{2 k}$ where there is at least one element of $\left\{e_{i_{1}}, \ldots, e_{i_{2 k}}\right\} \cap N_{p}$ in the set $\left\{x_{1}, \ldots, x_{2 k}\right\}$. Consequently

$$
\left\langle R_{2 k} \circ R_{2 k}\left(e_{i_{1}} \wedge \cdots \wedge e_{i_{2 k}}\right), e_{i_{2 k+1}} \wedge \cdots \wedge e_{i_{4 k}}\right\rangle=0 .
$$

Corollary 2.6. $P_{k}=0$ for $k \geqslant \frac{1}{2}(q+n-\mu)$.

PRoof. Let $q=2 p$, and choose $e_{1}, \ldots, e_{n}$ as above. If $\mu=n$ then we are done by Proposition 2.5. Now if $2 p>n-\mu$, then any $2 p$ vectors from the set $\left\{e_{1}, \ldots, e_{n}\right\}$ must contain an element from $N_{p}$ and it follows that $R_{2 p}=c I$ and $\mu=n$. We may assume then that $2 p \leqslant n-\mu$. Write $k=j p+s, 0 \leqslant s<p$. Then $2 k=j(2 p)+2 s$ so that $r=2 s . k-r=k-2 s=j p-s=(j-1) p+(p-s)$. Now if $(n-\mu) / 4 \geqslant k-r$ then $(n-\mu) / 2 \geqslant(j-1) 2 p+2(p-s)$. If $j p+s=k$ $\geqslant p+\frac{1}{2}(n-\mu) \geqslant(2 j-1) p+2(p-s)$ then $s \geqslant(j-1) p+2(p-s)$, a contradiction unless $j=1$. But if $j=1$ we have $s \geqslant \frac{1}{2}(n-\mu) \geqslant p$ a contradiction. Therefore $k-r>(n-\mu) / 4$ and the result follows from Proposition 2.5.

Our next result will be concerned with immersed submanifolds of Euclidean space. Suppose $M=N_{1} \times N_{2}$ and $f_{i}: N_{i} \rightarrow \mathbf{R}^{k_{i}}$ immerses $N_{i}$ into $\mathbf{R}^{k_{i}}$ such that $N_{1}$ has a flat normal bundle. If $f=f_{1} \times f_{2}$ is the product immersion then (1) $I I(X, Y)$ $=0$ for $X$ tangent to $N_{1}, Y$ tangent to $N_{2}$ and (2) $R(X, Y) \xi=0$ for $X, Y$ tangent to $N_{1}, \xi$ normal to $M$, where $R$ denotes the curvature tensor in the normal bundle. Furthermore axiomatic properties of Pontrjagin classes imply $P_{k}=0$ for $k$ $>\frac{1}{4} \operatorname{dim} N_{2}$. We shall now generalize this result to manifolds which are not necessarily products. Suppose $M$ is a submanifold of Euclidean space.

Definition 2.4. Let $C_{p}$ denote the collection of subspaces $Q_{p}$ in $T_{p} M$ such that (1) $I I(X, Y)=0$ for $X \in Q_{p}, Y \in W_{p}$ where $W_{p}$ denotes the subspace orthogonal to $Q_{p}$ and (2) $R(X, Y) \xi=0$ for $X, Y \in W_{p}, \xi$ normal to $M$ at $p$.

Note that $T_{p} M$ is always an element of $C_{p}$.

DeFINITION 2.5. (a) $\mu_{p}=\min \left\{\operatorname{dim} Q_{p}: Q_{p} \in C_{p}\right\}$.

(b) $\mu=\sup _{p} \mu_{p}$.

Proposition 2.7. $P_{k}=0$ for $k>\frac{1}{4} \mu$.

Proof. Let $N_{1}, \ldots, N_{p}$ be a local orthonormal frame of normal vectors to $M$ with $A_{1}, \ldots, A_{p}$ locally defined symmetric tensors of type $(1,1)$ satisfying $\left\langle A_{j}(X), Y\right\rangle=\left\langle I I(X, Y), N_{j}\right\rangle$. Locally $R_{2 k}=2^{-k}\left(\sum A_{j}^{2}\right)^{k}$ where the powers denote star multiplication. Fix a point $x \in M$ and choose a subspace $Q_{x} \in C_{x}$ such that $\operatorname{dim} Q_{x}=\mu_{x}$. If $X \in Q_{x}, Y \in W_{x}$ then $0=I I(X, Y)=\Sigma\left\langle A_{j}(X), Y\right\rangle N_{j}$ so that both $Q_{x}$ and $W_{x}$ are invariant under $A_{j}, j=1,2, \ldots, p$. The Ricci equations imply that $A_{1}, \ldots, A_{p}$ commute when restricted to $W_{x}$. Consequently there exists an orthonormal basis, $e_{1}, \ldots, e_{r}$, for $W_{x}$ such that $e_{i}$ is an eigenvector for $A_{j}$, $i=1,2, \ldots, r, j=1,2, \ldots, p$. Extend this to an orthonormal basis $e_{1}, e_{2}, \ldots, e_{n}$ for $T_{x} M$. Then $\left\langle A_{j_{1}}\left(e_{i_{1}}\right), A_{j_{2}}\left(e_{i_{2}}\right)\right\rangle=0$ for $i_{1}<r$ and $i_{1} \neq i_{2}$. If $k>\frac{1}{4} \mu$ and $e_{i_{1}}$ $\wedge \cdots \wedge e_{i_{4 k}} \neq 0$ then at least one of the subscripts must be $r$ or less. In this case it is routine to verify that

$$
F_{2 k}\left(A_{s_{1}} * A_{s_{2}} * \cdots * A_{s_{2 k}}, A_{j_{1}} * A_{j_{2}} * \cdots * A_{j_{2 k}}\right)\left(e_{i_{i}}, e_{i_{2}}, \ldots, e_{i_{4 k}}\right)=0
$$

and therefore $F_{2 k}\left(R_{2 k}, R_{2 k}\right)=0$. 
REMARK. Proposition 2.7 gives a direct proof of the fact that $P_{k}=0, k \geqslant 1$, for a submanifold of Euclidean space having a flat normal bundle.

3. Linear polynomial operators. Let $M$ be an $n$-dimensional Riemannian manifold. Given a local orthonormal frame $E=\left\{e_{1}, \ldots, e_{n}\right\}$, denote by $\Theta=$ $\left\{\theta^{1}, \ldots, \theta^{n}\right\}$ the dual coframe. If $I=\left(i_{1}, \ldots, i_{k}\right), 1<i_{j} \leqslant n$, is a multi-index let $e_{I}=e_{i_{1}} \wedge \cdots \wedge e_{i_{k}}, \Theta^{I}=\theta^{i_{1}} \wedge \cdots \wedge \theta^{i_{k}}$. By a polynomial in the components of the curvature tensor, $R$, we shall mean a real polynomial in the $n^{4}$ variables $z_{i j k l}$. If $P$ is a polynomial in the components of $R$ then $P\left(R_{E}\right)$ will denote the function given by setting $z_{i j k l}$ equal to $R_{i j k l}=\left\langle R\left(e_{i}, e_{j}\right) e_{k}, e_{l}\right\rangle$.

Definition 3.1. An element, $A$, of $\Gamma\left[\right.$ End $\left.\Lambda^{k}(T M)\right], k \geqslant 1$, will be called a "linear polynomial operator" provided there exist homogeneous linear polynomials, $C_{I J}$, in the components of $R$ such that with respect to any local orthonormal frame, $E=\left\{e_{1}, \ldots, e_{n}\right\}, A=\Sigma C_{I J}\left(R_{E}\right) \Theta^{I} \otimes e_{J}$.

As one example we have the curvature operator, $R_{2} . R_{2}$ may be written locally as $\frac{1}{4} \sum R_{i j k l} \theta^{i} \wedge \theta^{j} \otimes e_{k} \wedge e_{l}$. Define tensors $T_{1}, T_{2}$ of type $(1,1)$ on $M$ by $\left\langle T_{1}(X), Y\right\rangle=\operatorname{Ric}(X, Y)$, and $T_{2}=\tau I$ where Ric denotes the Ricci tensor and $\tau$ denotes the scalar curvature. It is easy to check that $T_{1}$ and $T_{2}$ are linear polynomial operators. Furthermore if $A$ is a linear polynomial operator in $\Gamma\left[\right.$ End $\left.\Lambda^{k}(T M)\right]$ and $I_{j}$ denotes the identity element of $\Gamma\left[\right.$ End $\Lambda^{j}(T M)$ ] then $A * I_{j}$ is a linear polynomial operator in $\Gamma\left[\right.$ End $\left.\Lambda^{k+j}(T M)\right]$. The following result characterizes linear polynomial operators.

THEOREM 3.1. Let $B$ be a linear polynomial operator in $\Gamma\left[\right.$ End $\left.\Lambda^{k}(T M)\right]$.

(a) If $k=1, B=a T_{1}+b T_{2}, a, b \in \mathbf{R}$.

(b) If $k \geqslant 2, B=\left(a T_{1}+b T_{2}\right) * I_{k-1}+c R_{2} * I_{k-2}, a, b, c \in \mathbf{R}$.

The proof of Theorem 3.1 will take a bit of time and discussion. Let $V$ denote $\mathbf{R}^{n}$ with the usual inner product and let $U=\left\{U_{j}\right\}$ be the standard basis with dual basis $U^{*}=\left\{U_{j}^{*}\right\} . O(n)$ acts on $V$ in the usual way and on $V^{*}$ by $g w(v)=w\left(g^{-1} v\right)$, $g \in O(n), w \in V^{*}, v \in V$. The action of $O(n)$ on the tensor algebra is defined by $g(v \otimes w)=g v \otimes g w$. Given a linear polynomial operator $A \in \Gamma\left[\right.$ End $\left.\Lambda^{k}(T M)\right]$ define a linear mapping

$$
L: \stackrel{4}{\otimes} V^{*} \rightarrow \text { End } \Lambda^{k} V \text { by } L(S)=\sum C_{I J}(S)\left(U^{*}\right)^{I} \otimes U_{J},
$$

where the polynomials $C_{I J}$ are evaluated on the components of $S$ with respect to the standard basis. An orthonormal basis $e_{1}, \ldots, e_{n}$ for $T_{p} M$ determines an isomorphism of $T_{p} M$ with $V$ given by $e_{j} \leftrightarrow U_{j}$. Under this identification $R$ is an element of $\otimes^{4} V^{*}$ and $A=L(R)$. Furthermore the definition of a linear polynomial operator implies that $g^{-1}(L(g R))=L(R)$ for all $g \in O(n)$. Let $d g$ denote normalized Haar measure on $O(n)$ and define $\bar{L}=\int_{O(n)} g^{-1} L g d g$. Then $g^{-1} \bar{L} g=$ $\bar{L}$ and $\bar{L}(R)=A$. Thus to find $A$ it suffices to determine

$$
\begin{aligned}
& \operatorname{Hom}_{O(n)}\left(\stackrel{4}{\otimes} V^{*}, \text { End } \Lambda^{k} V\right) \\
& =\left\{L \in \operatorname{Hom}\left(\stackrel{4}{\otimes} V^{*}, \text { End } \Lambda^{k} V\right): g^{-1} L g=L, g \in O(n)\right\} .
\end{aligned}
$$


Let $N$ and $k$ be fixed positive integers and define $P_{k} \in \operatorname{End}\left[\otimes^{N+k} V^{*}\right]$ to be alternation over the last $k$ indices. Given $\sigma \in S_{N+k}$ we let $r(\sigma)$ denote the number of integers $j>N$ such that $\sigma(j) \leqslant N$. For $N$ even and $r \leqslant \min (N / 2, k)$ define $\alpha$, $\alpha_{r} \in \operatorname{Hom}_{O(n)}\left(\otimes^{N+k} V^{*}, \Lambda^{k} V\right)$ in terms of standard components by

$$
\alpha: W_{L_{1} L_{2} \cdots L_{N+k}} \rightarrow W_{j_{1} j_{1} j_{2} j_{2} \cdots L_{j} j_{s}}^{\left.L_{j_{2}}\right]}
$$

( $N=2 s$, square brackets denote alternation) and $\alpha_{r}=\alpha \circ \tau_{r}$, where $\tau_{r}=$ $(2, N+1)(4, N+2) \cdots(2 r, N+r) \in S_{N+k}$ permutes the indices of $W_{L_{1} L_{2}} \cdots L_{N+k}$.

LEMMA 3.2. (a) $\operatorname{Hom}_{O(n)}\left(\otimes^{N+k} V^{*}, \Lambda^{k} V\right)$ is nonzero only for $N$ even and in this case is spanned by $\left\{\alpha_{\sigma} \equiv \alpha \circ \sigma, \sigma \in S_{N+k}\right\}$,

(b) for $N$ even, $\alpha_{\sigma} \circ P_{k}=0$ if $r(\sigma)>N / 2$,

(c) for $N$ even, if $\alpha_{\sigma} \circ P_{k} \neq 0$ then there exists $\tau \in S_{N+k}$ such that $\tau(j) \leqslant N$ for $j \leqslant N$ and $\alpha_{\sigma}= \pm \alpha_{r} \circ \tau$, where $r=r(\sigma)$.

PROOF. (a) is essentially the corollary on p. 286 of [1]. The proofs of (b) and (c) are routine and will be left to the reader.

There exists a mapping $G: A \rightarrow \mathscr{Q}$ from $\operatorname{Hom}_{O(n)}\left(\bigotimes^{N} V^{*}\right.$, End $\left.\Lambda^{k} V\right)$ to $\operatorname{Hom}_{O(n)}\left(\otimes^{N+k} V^{*}, \Lambda^{k} V\right)$ defined by

$$
\mathbb{Q}\left(w_{1} \otimes w_{2} \otimes \cdots \otimes w_{N+k}\right)=\left[A\left(w_{1} \otimes \cdots \otimes w_{N}\right)\right]\left(w_{N+1} \wedge \cdots \wedge w_{N+k}\right) .
$$

It is clear that this mapping is injective and that $\mathscr{Q}=\mathscr{Q} \circ P_{k}$. For $N$ even and $0 \leqslant r \leqslant N / 2$ define $A_{r} \in \operatorname{Hom}_{O(n)}\left(\otimes^{N} V^{*}\right.$, End $\left.\Lambda^{r} V\right)$ by

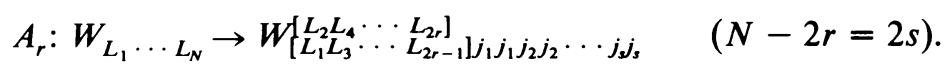

If $r \leqslant k$ we may define $A_{r} * I_{k-r}$ in $\operatorname{Hom}_{O(n)}\left(\otimes^{N} V^{*}\right.$, End $\left.\Lambda^{k} V\right)$ by $A_{r} * I_{k-r}(W)=$ $A_{r}(W) * I_{k-r}$ where $I_{k-r}$ denotes the identity in End $\Lambda^{k-r} V$. Let $Q_{r} * \mathcal{G}_{k-r}$ denote the image of $A_{r} * I_{k-r}$ under the mapping $A \rightarrow Q$. A routine computation shows that $\alpha_{r} \circ P_{k}$ is a multiple of $Q_{r} * \mathscr{G}_{k-r}$.

Proposition 3.3. Hom ${ }_{O(n)}\left(\otimes^{N} V^{*}\right.$, End $\left.\Lambda^{k} V\right)=0$ for $N$ odd and for $N$ even is generated by $\left\{\left(A_{r} * I_{k-r}\right) \circ \tau: 0 \leqslant r \leqslant \min (N / 2, k), \tau \in S_{N}\right\}$.

Proof. It follows from Lemma 3.2 that $\operatorname{Hom}_{O(n)}\left(\otimes^{N} V^{*}\right.$, End $\left.\Lambda^{k} V\right)$ is zero for $N$ odd. If $N$ is even and $A$ is in $\operatorname{Hom}_{O(n)}\left(\bigotimes^{N} V^{*}\right.$, End $\left.\Lambda^{k} V\right)$ then $Q=\sum a_{\sigma} \alpha_{\sigma}$ for some choice of constants $a_{\sigma}$. Furthermore $\mathbb{Q}=\mathbb{Q} \circ P_{k}=\sum a_{\sigma}\left(\alpha_{\sigma} \circ P_{k}\right)$. It will be shown that given $\alpha_{\sigma} \circ P_{k} \neq 0$ there exists $\tau \in S_{N}$ such that $\alpha_{\sigma} \circ P_{k}$ is a multiple of the image of $\left(A_{r} * I_{k-r}\right) \circ \tau(r=r(\sigma))$ under the mapping $G$. The injectivity of $G$ will then imply the result.

If $\alpha_{\sigma} \circ P_{k} \neq 0$ there exists $\tau^{\prime} \in S_{N+k}$ such that $\alpha_{\sigma}= \pm \alpha_{r} \circ \tau^{\prime}(r=r(\sigma))$ and $\tau^{\prime}=\tau \beta$ where $\tau$ fixes the integers $\{N+1, \ldots, N+k\}$ and $\beta$ fixes the integers $\{1,2, \ldots, N\}$. Now $\tau^{\prime} \circ P_{k}=\varepsilon(\beta) P_{k} \circ \tau$ so that

$$
\alpha_{\sigma} \circ P_{k}= \pm \sigma_{r} \circ \tau^{\prime} \circ P_{k}= \pm \sigma_{r} \circ P_{k} \circ \tau
$$

which is equal to a multiple of $Q_{r} * G_{k-r} \circ \tau$. Since $Q_{r} * G_{k-r} \circ \tau$ is equal to the image of $\left(A_{r} * I_{k-r}\right) \circ \tau$ under $G$ the result follows.

We now give the proof of Theorem 3.1. 
Proof. A generating set for $\operatorname{Hom}_{O(n)}\left(\otimes^{4} V^{*}\right.$, End $\left.\Lambda^{k} V\right)$ is given by $\left\{A_{r} * I_{k-r} \circ \tau: 0 \leqslant r \leqslant \min (2, k), \tau \in S_{4}\right\}$. By evaluating each of these elements on the curvature tensor $R$ we produce a generating set for the space of linear polynomial operators. The curvature identities greatly reduce the number of possibilities. $A_{0} \circ \tau(R)$ is always a multiple of the scalar curvature so that for $k \geqslant 1, A_{0} * I_{k} \circ \tau(R)$ is always a multiple of $T_{2} * I_{k-1} . A_{1} * I_{k-1} \circ \tau(R)$ is always a multiple of $T_{1} * I_{k-1}$. Finally $A_{2} * I_{k-2} \circ \tau(R)$ is always a multiple of $R_{2} * I_{k-2}$ and the result follows.

We now use Theorem 3.1 to derive the decomposition of the curvature operator given in [8]. Define an inner product (, ) on End $\Lambda^{2} V$ by the formula $(A, B)=$ trace $B^{t} \circ A$. With this inner product $O(n)$ acts naturally on End $\Lambda^{2} V$ as orthogonal transformations. Let $S_{1}$ denote the orthogonal complement to the kernel of trace: End $\Lambda^{2} V \rightarrow \mathbf{R}$. Since $\operatorname{dim} S_{1}=1$ and the identity $I_{2}$ is in $S_{1}$ it follows that $S_{1}$ consists of all scalar multiples of $I_{2}$ and is thus $O(n)$ invariant. If $\left\{e_{L}\right\}$ is an orthonormal basis for $V$ we may define the "Ricci contraction" Ric: End $\Lambda^{2} V \rightarrow$ End $V$ by

$$
\langle(\text { Ric } B) X, Y\rangle=\Sigma\left\langle B\left(X \wedge e_{j}\right), Y \wedge e_{j}\right\rangle .
$$

$S_{2}=\operatorname{Ker} \Re i c$ is contained in Ker trace and since $\mathcal{R} i c$ is $O(n)$ equivariant $S_{2}$ is an $O(n)$ invariant subspace of End $\Lambda^{2} V$. Finally if we define $S_{3}$ to be the intersection of the kernel of trace with the orthogonal complement to $S_{2}$ then we may write End $\Lambda^{2} V$ as an orthogonal direct sum of $O(n)$ invariant subspaces

$$
\text { End } \Lambda^{2} V=S_{1} \oplus S_{2} \oplus S_{3} \text {. }
$$

The choice of an orthonormal basis for $T_{p} M$ allows us to identify $T_{p} M$ and $V$ and realize the curvature tensor $R$ and the curvature operator $R_{2}$ as elements of $\otimes^{4} V^{*}$ and End $\Lambda^{2} V$ respectively. We wish to determine the $S_{L}$ component of $R_{2}$ for $L=1,2,3$. Since $I_{2}$ spans $S_{1}$ the $S_{1}$ component of $R_{2}$ is easy to find and is simply

$$
\frac{\left(R_{2}, I_{2}\right)}{\left(I_{2}, I_{2}\right)} I_{2}=\frac{\tau}{n(n-1)} I_{2} \text {. }
$$

It is less clear how to find the $S_{2}$ component and it is here that we invoke Theorem 3.1. Choose an element $L$ of $\operatorname{Hom}_{O(n)}\left(\otimes^{4} V^{*}\right.$, End $\left.\Lambda^{2} V\right)$ such that $L(R)=R_{2}$ and let $\pi$ : End $\Lambda^{2} V \rightarrow S_{2}$ denote orthogonal projection. Since $S_{2}$ is $O(n)$ invariant $\pi \circ L$ is also an element of $\operatorname{Hom}_{O(n)}\left(\otimes^{4} V^{*}\right.$, End $\left.\Lambda^{2} V\right)$ and thus $\pi\left(R_{2}\right)=(\pi \circ L) R$ is the value of a linear polynomial operator at $p \in M$. Now $\operatorname{Ric}\left(R_{2}\right)=T_{1}, \operatorname{Ric}\left(T_{1} * I\right)=T_{2}+(n-2) T_{1}$, and $\operatorname{Ric}\left(T_{2} * I\right)=2(n-1) T_{2}$. Since $\Re i c\left(\pi\left(R_{2}\right)\right)=0$ it follows from Theorem 3.1 that $\pi\left(R_{2}\right)$ is a multiple of

$$
C=R_{2}-\frac{1}{n-2} T_{1} * I+\frac{1}{2(n-1)(n-2)} T_{2} * I .
$$

$C$ is called the Weyl curvature operator and for $n \geqslant 4$ vanishes identically if and only if $M$ is conformally flat [5]. Thus $\pi\left(R_{2}\right)=\left(R_{2}, C\right) C /(C, C)=C$. The $S_{3}$ component of $R_{2}$ is then easily computed to be

$$
B=R_{2}-\frac{\tau}{n(n-1)} I_{2}-C=\frac{1}{n-2}\left(T_{1} * I-\frac{T_{2} * I}{n}\right) .
$$


$B$ is the so-called traceless Ricci tensor and for $n \geqslant 3, B$ vanishes identically if and only if $M$ is Einstein.

One further comment may be made with respect to this decomposition of the curvature operator. It follows from Lemma 2.1(a) that $F_{2 k}\left(R_{2 k}, R_{2 k}\right)=$ $F_{2 k}\left(C^{k}, C^{k}\right)$ where $C^{k}=C * C * \cdots * C$ (k times). Thus the Weyl curvature operator contains all the information necessary to construct the Pontrjagin classes.

\section{REFERENCES}

1. M. Atiyah, R. Bott and V. K. Patodi, On the heat equation and the index theorem, Invent. Math. 19 (1973), 279-330.

2. S. S. Chern, On the curvature and characteristic classes of a Riemannian manifold, Abh. Math. Sem. Univ. Hamburg 20 (1956), 117-126.

3. S. S. Chern and N. H. Kuiper, Some theorems on the isometric imbedding of compact Riemann manifolds in Euclidean space, Ann. of Math. (2) 56 (1952), 422-430.

4. Y. K. Cheung and C. C. Hsiung, Curvature and characteristic classes of compact Riemannian manifolds, J. Differential Geom. 1 (1967), 89-97.

5. L. P. Eisenhart, Riemannian geometry, Princeton Univ. Press, Princeton, N. J., 1949.

6. A. Gray, Some relations between curvature and characteristic classes, Math. Ann. 184 (1969), 257-267.

7. R. S. Kulkarni, On the Bianchi identities, Math. Ann. 199 (1972), 175-204.

8. I. M. Singer and J. A. Thorpe, The curvature of 4-dimensional Einstein spaces, Global Analysis, Papers in Honor of K. Kodaira (D. C. Spencer and S. Iyanaga, editors), Princeton Univ. Press, Princeton, N. J., 1969, pp. 355-365.

9. A. Stehney, Courbure d'ordre $p$ et les classes de Pontrjagin, J. Differential Geom. 8 (1973), $125-133$.

10. J. Thorpe, Sectional curvature and characteristic classes, Ann. of Math. (2) 80 (1964), 429-443.

Department of Mathematics, Rice University, Houston, TeXas 77001 